\title{
Preconditioners for High Order Mortar Methods based on Substructuring
}

\author{
Silvia Bertoluzza ${ }^{1}$ and Micol Pennacchio ${ }^{1}$ \\ Istituto di Matematica Applicata e Tecnologie Informatiche - C.N.R., via Ferrata, \\ 1 - 27100 Pavia, Italy. SSilvia.Bertoluzza, Micol.Pennacchio\}@imati.cnr.it
}

\begin{abstract}
Summary. A class of preconditioners for the Mortar Method based on substructuring is studied. We generalize the results of Achdou, Maday and Widlund (AMW99), obtained for the case of order one finite elements, to a wide class of discretization spaces including finite elements of any orders. More precisely, we show that the condition number of the preconditioned matrix grows at most polylogarithmically with the number of degrees of freedom per subdomain.
\end{abstract}

\section{Introduction}

We deal with the Mortar method, a nonconforming version of domain decomposition methods, that allows different discretizations and/or methods in different subdomains. Consequently, in an adaptive strategy, refinement can be carried out in each subdomain independently and it is possible to use in each subdomain the best suited method.

Here we face the problem of the efficient solution of the linear system arising from such discretization in order to make these techniques more competitive for real life applications. After elimination of the degrees of freedom internal to the subdomains, we need to find the traces of the solution on the subdomain boundaries, i.e. to solve the Schur complement system. The approach that we follow is the substructuring one, proposed by Bramble, Pasciak and Schatz (BPS86) in the framework of conforming domain decomposition. Such an approach was already applied to the Mortar method by Achdou, Maday and Widlund in (AMW99) for the case of order one finite elements. This consists in considering a suitable splitting of the nonconforming discretization space in terms of "edge" and "vertex" degrees of freedom and then using the related block-Jacobi type preconditioners. In this work we generalize the results of (AMW99) to a wide class of discretization spaces (including finite elements of any order) showing that the condition number of the preconditioned matrix grows at most polylogarithmically with the number of degrees of freedom per subdomain, analogously to what happens for the order one case. 
Finally, we present numerical tests showing the scalability of the method for $Q_{1}$ and $Q_{2}$ finite elements.

\section{The Mortar Method}

At first we briefly introduce the Mortar method with its main properties (see (BMP94; W01)) and we focus, for simplicity, on the following simple model problem, even if the results of this paper can be easily extended to a more general situation. Let $\Omega \subset \mathbb{R}^{2}$ be a polygonal domain and $f \in L^{2}(\Omega)$, then we look for $u$ satisfying

$$
-\sum_{i, j=1}^{2} \frac{\partial}{\partial \mathbf{x}_{j}}\left(a_{i j}(\mathbf{x}) \frac{\partial u}{\partial \mathbf{x}_{i}}\right)=f \text { in } \Omega, \quad u=0 \text { on } \partial \Omega .
$$

The matrix $a(\mathbf{x})=\left(a_{i j}(\mathbf{x})\right)_{i, j=1,2}$ is assumed to be, for almost all $\mathbf{x} \in \Omega$, symmetric positive definite with smallest eigenvalue $\geq \alpha>0$ and largest eigenvalue $\leq \alpha^{\prime}, \alpha, \alpha^{\prime}$ independent of $\mathbf{x}$.

In order to solve (1) we decompose the computational domain $\Omega$ as the union of $L$ subdomains $\Omega_{\ell}, \Omega=\bigcup_{\ell=1, \ldots, L} \Omega_{\ell}$, which, for the sake of simplicity we assume to be quadrilateral (in general the constants in the inequalities will depend on the number of edges of the subdomains as well as on their aspect ratio). We follow the notation of (BP01): we set

$$
\Gamma_{\ell n}=\partial \Omega_{n} \cap \partial \Omega_{\ell}, \quad \mathcal{S}=\cup \Gamma_{\ell n}
$$

and we denote by $\gamma_{\ell}^{(i)}(i=1, \ldots, 4)$ the $i$-th side of the $\ell$-th domain so that $\partial \Omega_{\ell}=\bigcup_{i=1}^{4} \gamma_{\ell}^{(i)}$.

Definition 1. We say that a decomposition is geometrically conforming if each edge $\gamma_{\ell}^{(i)}$ coincides with $\Gamma_{\ell n}$ for some $n$. If the decomposition is not geometrically conforming, then each interior edge $\gamma_{\ell}^{(i)}$ will be in general split as the union of several segments $\Gamma_{\ell n}$ :

$$
\gamma_{\ell}^{(i)}=\bigcup_{n \in I_{\ell}^{(i)}} \Gamma_{\ell n}
$$

where $I_{\ell}^{(i)}=\left\{n:\left|\partial \Omega_{n} \cap \gamma_{\ell}^{(i)}\right| \neq 0\right\}$.

We now consider a non conforming domain decomposition method, based on the above splitting of the domain $\Omega$, for the solution of problem (1). First we introduce the corresponding functional setting, hence let

$$
X=\prod_{\ell}\left\{u_{\ell} \in H^{1}\left(\Omega_{\ell}\right) \mid u_{\ell}=0 \text { on } \partial \Omega \cap \partial \Omega_{\ell}\right\}, \quad T=\prod_{\ell} H_{*}^{1 / 2}\left(\partial \Omega_{\ell}\right)
$$


with $H_{*}^{1 / 2}\left(\Omega_{\ell}\right)=H^{1 / 2}\left(\partial \Omega_{\ell}\right)$ if $\partial \Omega_{\ell} \cap \partial \Omega=\emptyset$ and $H_{*}^{1 / 2}\left(\partial \Omega_{\ell}\right)=\{\eta \in$ $\left.H^{1 / 2}\left(\partial \Omega_{\ell}\right),\left.\eta\right|_{\partial \Omega_{\ell} \cap \partial \Omega} \equiv 0\right\} \sim H_{00}^{1 / 2}\left(\partial \Omega_{\ell} \backslash \partial \Omega\right)$ otherwise. We denote by $\|\cdot\|_{1 / 2, \partial \Omega_{\ell}}$ the related norm, and by $\|\cdot\|_{-1 / 2, \ell}$ the norm of the corresponding dual space.

On $X$ and $T$ we introduce the following broken norm and semi-norm: $\|u\|_{X}=\left(\sum_{\ell}\|u\|_{1, \Omega_{\ell}}^{2}\right)^{1 / 2},|u|_{X}=\left(\sum_{\ell}|u|_{1, \Omega_{\ell}}^{2}\right)^{1 / 2},\|\eta\|_{T}=\left(\sum_{\ell}\left\|\eta_{\ell}\right\|_{1 / 2, \partial \Omega_{\ell}}^{2}\right)^{1 / 2}$.

For each $\ell$, let $\mathcal{V}_{h}^{\ell}$ be a family of finite dimensional subspaces of $H^{1}\left(\Omega_{\ell}\right) \cap$ $C^{0}\left(\bar{\Omega}_{\ell}\right)$, depending on a parameter $h=h_{\ell}>0$ and satisfying an homogeneous boundary condition on $\partial \Omega \cap \partial \Omega_{\ell}$.

Let $T_{h}^{\ell}=\left.\mathcal{V}_{h}^{\ell}\right|_{\partial \Omega_{\ell}}$, and, for each edge $\gamma_{\ell}^{(i)}$ of the subdomain $\Omega_{\ell}$,

$$
\begin{gathered}
T_{\ell, i}=\left\{\eta: \eta \text { is the trace on } \gamma_{\ell}^{(i)} \text { of some } u_{\ell} \in \mathcal{V}_{h}^{\ell}\right\} \\
T_{\ell, i}^{0}=\left\{\eta \in T_{\ell, i}: \eta=0 \text { at the vertices of } \gamma_{\ell}^{(i)}\right\} .
\end{gathered}
$$

We set

$$
X_{h}=\prod_{\ell=1}^{L} \mathcal{V}_{h}^{\ell} \subset X, \quad T_{h}=\prod_{\ell=1}^{L} T_{h}^{\ell} \subset T
$$

and we define a composite bilinear form $a_{X}: X \times X \longrightarrow \mathbb{R}$ as follows:

$$
a_{X}(u, v)=\sum_{\ell} \int_{\Omega_{\ell}} \sum_{i, j} a_{i j}(\mathbf{x}) \frac{\partial u_{\ell}}{\partial \mathbf{x}_{i}} \frac{\partial v_{\ell}}{\partial \mathbf{x}_{j}} d \mathbf{x}
$$

The bilinear form $a_{X}$ is clearly not coercive on $X$. In order to obtain a well posed problem, we will then consider proper subspaces of $X$ consisting of functions that satisfy a suitable weak continuity constraint defined, according to the Mortar method, by choosing a splitting of the skeleton $\mathcal{S}$ as the disjoint union of a certain number of subdomain sides $\gamma_{\ell}^{(i)}$ called "multiplier sides". We denote by $I \subset\{1, \ldots, L\} \times\{1, \ldots, 4\}$ the set of indices $(l, i)$ such that $\gamma_{l}^{(i)}$ is a multiplier side, while $I^{*} \subset\{1, \cdots, L\} \times\{1, \cdots, 4\}$ will denote the indexset corresponding to "trace sides" ("mortars" or "master sides" in the usual terminology).

For each $m=(\ell, i) \in I$ let a finite dimensional multiplier space $M_{h}^{m}$ (also depending on the parameter $h$ ) on $\gamma_{m}$,

$$
M_{h}^{m} \subset L^{2}\left(\gamma_{m}\right), \quad \operatorname{dim}\left(M_{h}^{m}\right)=\operatorname{dim}\left(T_{h}^{m, 0}\right),
$$

be given. We set:

$$
M_{h}=\left\{\eta \in H^{-1 / 2}(\mathcal{S}),\left.\forall m \in I \quad \eta\right|_{\gamma_{m}} \in M_{h}^{m}\right\} \sim \prod_{m \in \mathrm{i}} M_{m} .
$$


The constrained approximation and trace spaces $\mathcal{X}_{h}$ and $\mathcal{T}_{h}$ are then defined as follows:

$$
\begin{gathered}
\mathcal{X}_{h}=\left\{v_{h} \in X_{h}, \int_{S}\left[v_{h}\right] \lambda d s=0, \forall \lambda \in M_{h}\right\} \\
\mathcal{T}_{h}=\left\{\eta \in T_{h}, \int_{S}[\eta] \lambda d s=0, \forall \lambda \in M_{h}\right\} .
\end{gathered}
$$

The elements of $\mathcal{X}_{h}$ can be obtained by applying to any element of $X_{h}$ a correction operator $\mathcal{P}_{h}: X_{h} \rightarrow \mathcal{X}_{h}$, whose action consists in suitably modify its argument to impose the constraint; remark that $\mathcal{P}_{h}$ is a projector.

Thus we can introduce the following discrete problem:

Problem 1. Find $u_{h} \in \mathcal{X}_{h}$ such that for all $v_{h} \in \mathcal{X}_{h}$

$$
a_{X}\left(u_{h}, v_{h}\right)=\int_{\Omega} f v_{h}
$$

It is not difficult to choose the class $M_{h}$ of multipliers in such a way to guarantee ellipticity uniformly with respect to the mesh-size parameter $h$ and to the number $L$ of subdomains.

Then it can be proved that for all $h>0$, Problem (1) admits a unique solution $u_{h}$ which satisfies the following error estimate (BP01):

$$
\left\|u-u_{h}\right\|_{X} \lesssim\left(\inf _{v_{h} \in \mathcal{X}_{h}}\left\|u-v_{h}\right\|_{X}+\inf _{\lambda \in M_{h}}\left\|\frac{\partial u}{\partial \nu}-\lambda\right\|_{-1 / 2, \mathcal{S}}\right)
$$

with $\|\cdot\|_{-1 / 2, \mathcal{S}}$ denoting the norm of $T^{\prime}$, dual of $T$.

\section{Substructuring Preconditioners for the Mortar Element Method}

In this section we focus on a class of preconditioners for the linear system arising from the discretization by the Mortar method. We will follow the "substructuring" approach first introduced in (BPS86) and already studied in the case of the Mortar Finite Element method in (AMW99). The main idea of these preconditioners consists in distinguishing three types of degrees of freedom: interior degrees of freedom (corresponding to basis functions vanishing on the skeleton and supported on one sub-domain), edge degrees of freedom, and vertex degrees of freedom. Consequently we can split the functions $u \in \mathcal{X}_{h}$ as the sum of three suitably defined components: $u=u^{0}+u^{E}+u^{V}$. Moreover, when expressed in a basis related to such a splitting, substructuring preconditioners can be written in a block diagonal form. 
Let us now examine in details how the splitting is constructed. Given any discrete function $w=\left(w_{\ell}\right)_{\ell=1, \cdots, L} \in X_{h}$ we can split it in a unique way as the sum of an interior function $w^{0} \in \mathcal{X}_{h}^{0}$ and a discrete lifting, performed subdomainwise of its trace $\eta(w)=\left(\left.w^{\ell}\right|_{\Omega_{\ell}}\right)_{\ell=1, \cdots, L}$ which by abuse of notation we will denote by $R_{h}(w)$ (rather than using the heavier notation $R_{h}(\eta(w))$ ):

$$
w=w^{0}+R_{h}(w), \quad w^{0} \in \mathcal{X}_{h}^{0}
$$

with $R_{h}(w)=\left(R_{h}^{\ell}\left(w_{\ell}\right)\right)_{\ell=1, \ldots, K}, R_{h}^{\ell}\left(w_{\ell}\right)$ being the unique element in $\mathcal{V}_{h}^{\ell}$ satisfying

$$
\begin{aligned}
R_{h}^{\ell}\left(w_{\ell}\right) & =w_{\ell} & \text { on } \Gamma_{\ell} \\
\int_{\Omega_{\ell}} \sum_{i, j} a(\mathbf{x}) \frac{\partial}{\partial \mathbf{x}_{i}} \frac{\partial}{\partial \mathbf{x}_{j}} R_{h}^{\ell}\left(w_{\ell}\right) v_{h}^{\ell} d \mathbf{x} & =0, & \forall v_{h} \in \mathcal{V}_{h}^{\ell} \cap H_{0}^{1}\left(\Omega_{\ell}\right) .
\end{aligned}
$$

Thus the spaces $X_{h}$ of unconstrained functions and $\mathcal{X}_{h}$ of constrained functions can be split as direct sums of an interior and of a (respectively unconstrained or constrained) trace component:

$$
X_{h}=X_{h}^{0} \oplus R_{h}\left(T_{h}\right), \quad \mathcal{X}_{h}=\mathcal{X}_{h}^{0} \oplus R_{h}\left(\mathcal{T}_{h}\right) .
$$

We can easily verify that $a_{X}: X_{h} \times X_{h} \rightarrow \mathbb{R}$ satisfies

$$
a_{X}(w, v)=a_{X}\left(w^{0}, v^{0}\right)+a_{X}\left(R_{h}(w), R_{h}(v)\right):=a_{X}\left(w^{0}, v^{0}\right)+s(\eta(w), \eta(v)),
$$

where the discrete Steklov-Poincaré operator $s: T_{h} \times T_{h} \rightarrow \mathbb{R}$ is defined by

$$
s(\xi, \eta):=\sum_{\ell} \int_{\Omega_{\ell}}\left(a(\mathbf{x}) \nabla R_{h}^{\ell}(\xi)\right) \cdot \nabla R_{h}^{\ell}(\eta) .
$$

We note that the problem of preconditioning the matrix $A$ associated to the discretization of $a_{X}$, reduces to finding good preconditioners for the matrices $A_{0}$ and $S$ corresponding respectively to the bilinear forms $a_{X}: \mathcal{X}_{h}^{0} \times \mathcal{X}_{h}^{0} \longrightarrow \mathbb{R}$ and $s: T_{h} \times T_{h} \longrightarrow \mathbb{R}$. The matrix $A_{0}$ is block diagonal since the coupling between subdomains is taken into account only by the Steklov-Poincaré operator. The blocks of $A_{0}$ (which are in fact stiffness matrices corresponding to standard Dirichlet solvers) are widely studied in the literature; here we concentrate only on the preconditioning of the discrete Steklov-Poincaré operator $S$.

\subsection{The splitting of the trace space}

The space of constrained skeleton functions $\mathcal{T}_{h}$ defined in (6) can be further split as the sum of vertex and edge functions. More specifically, if we denote by $\mathfrak{L} \subset \prod_{\ell=1}^{L} H_{*}^{1 / 2}\left(\partial \Omega_{\ell}\right)$ the space 


$$
\mathfrak{L}=\left\{\left(\eta_{\ell}\right)_{\ell=1, \cdots, L}, \eta_{\ell} \text { is linear on each edge of } \Omega_{\ell}\right\},
$$

then we can define the space of constrained vertex functions as

$$
\mathcal{T}_{h}^{V}=\mathcal{P}_{h} \mathfrak{L}
$$

with $\mathcal{P}_{h}$ the correction operator imposing the constraint. In the following we will make the (not restrictive) assumption $\mathfrak{L} \subset T_{h}$, which yields $\mathcal{T}_{h}^{V} \subset \mathcal{T}_{h}$. by

We then introduce the space of constrained edge functions $\mathcal{T}_{h}^{E} \subset \mathcal{T}_{h}$ defined

$$
\mathcal{T}_{h}^{E}=\left\{\eta=\left(\eta_{\ell}\right)_{\ell=1, \cdots, L} \in \mathcal{T}_{h}, \eta_{\ell}(A)=0, \forall \text { vertex } A \text { of } \Omega_{\ell}\right\}
$$

and it is quite simple to check that a function in $\mathcal{T}_{h}^{E}$ is uniquely defined by its value on trace edges, the value on multiplier edges being forced by the constraint.

Thus, it can be easily verified that

$$
\mathcal{T}_{h}=\mathcal{T}_{h}^{V} \oplus \mathcal{T}_{h}^{E}
$$

and that each $\eta \in \mathcal{T}_{h}$ can be decomposed in a unique way as

$$
\eta=\eta^{V}+\eta^{E}, \quad \text { with } \quad \eta^{V} \in \mathcal{T}_{h}^{V} \text { and } \eta^{E} \in \mathcal{T}_{h}^{E} .
$$

\section{The preconditioner}

The preconditioner that we consider for $S$ is of block-Jacobi type with blocks related to edges and vertexes. More specifically we can assemble the preconditioner $\hat{s}$ as

$$
\begin{aligned}
& \hat{s}: \mathcal{T}_{h} \times \mathcal{T}_{h} \longrightarrow \mathbb{R} \\
& \hat{s}(\eta, \xi)=b^{V}\left(\eta^{V}, \xi^{V}\right)+b^{E}\left(\eta^{E}, \xi^{E}\right)
\end{aligned}
$$

with

$$
b^{V}: \mathcal{T}_{h}^{V} \times \mathcal{T}_{h}^{V} \longrightarrow \mathbb{R} \quad \text { such that } \quad b^{V}\left(\eta^{V}, \eta^{V}\right) \simeq s\left(\eta^{V}, \eta^{V}\right)
$$

and

$$
b^{E}: \mathcal{T}_{h}^{E} \times \mathcal{T}_{h}^{E} \longrightarrow \mathbb{R} \quad b^{E}(\eta, \xi)=\sum_{(\ell, i) \in I^{*}} b_{\ell, i}\left(\eta_{\ell}, \xi_{\ell}\right)
$$

where for any trace side $\gamma_{\ell}^{(i)},(\ell, i) \in I^{*}, b_{\ell, i}: T_{\ell, i}^{0} \times T_{\ell, i}^{0} \longrightarrow \mathbb{R}$ is a symmetric bilinear form satisfying for all $\eta \in T_{\ell, i}^{0}$

$$
b_{\ell, i}(\eta, \eta) \simeq\|\eta\|_{H_{00}^{1 / 2}\left(\gamma_{\ell}^{(i)}\right)} .
$$

Denoting by $H_{l}$ the diameter of $\Omega_{l}$ and writing conventionally $H / h=$ $\min _{l}\left\{H_{l} / h_{l}\right\}$ then, under suitable regularity assumptions on the subdomains and on the spaces considered (see (BP04)), we can prove the following theorem providing bounds for the condition number of the preconditioned matrix. 
Theorem 1. Let $S$ and $\hat{S}$ be the matrices obtained by discretizing respectively $s$ and $\hat{s}$. Then it holds

$$
\operatorname{Cond}\left(\hat{S}^{-1} S\right) \lesssim\left(1+\log \left(\frac{H}{h}\right)\right)^{4} .
$$

Moreover, if the decomposition is geometrically conforming then

$$
\operatorname{Cond}\left(\hat{S}^{-1} S\right) \lesssim\left(1+\log \left(\frac{H}{h}\right)\right)^{2} .
$$

The proof of Theorem 1 follows essentially the guidelines of the proofs of the analogous results in (BPS86; AMW99); due to space constraint, we do not present it but we refer to (BP04).

\section{Numerical tests}

Finally we performed numerical experiments to test the scalability of the method for $Q_{1}$ and $Q_{2}$ finite elements. The model problem is the Poisson equation on the unit square $\Omega$ with homogeneous Dirichlet boundary conditions. A uniform, geometrically conforming, decomposition of $\Omega$ in $K=N \times N$ equal square subdomains of size $H \times H$ with $H=1 / N$ is considered. In each subdomains $\Omega_{k}$, a uniform mesh $\mathcal{T}^{k}$ is built and $Q_{1}, Q_{2}$ finite elements are used in each square.

In order to study the dependence on $H$ (size of the subdomains) and on $h$ (finest meshsize of the finite element spaces), we set $n_{k}=n$ for all $k$; hence $h_{k}=h=H / n$ and $H / h=n$. This corresponds to a non-conforming implementation of the standard domain decomposition method. Then, we tested the preconditioners for several combinations of $N$ and $n$ with $n$ in the range $[5,40]$ and $N$ in the range $[4,32]$.

The preconditioned conjugate gradient iteration was stopped when the residual norm decreased by a factor of $10^{-5}$ and the experiment were carried out in MATLAB.

Tables 1 show the number of conjugate gradient iterations for reducing the residual of a factor $10^{-5}$ for $Q_{1}$ (left) and $Q_{2}$ (right) finite elements respectively. For the edge block of the preconditioner we considered the square root of the stiffness matrix associated to the discretization of the operator $-d^{2} / d x^{2}$ by $P_{1}$ and $P_{2}$ finite elements on each edge with homogeneous Dirichlet boundary conditions at the extrema.

The results are in close agreement with the theory: the condition number of the preconditioned matrix grows at most polylogarithmically with the number of degrees of freedom per subdomain, as indicated by theorem (1).

A complete set of numerical tests showing the scalability of the method for $Q_{1}$ and $Q_{2}$ finite elements can be found in (BP04). 


\begin{tabular}{c|cccc}
\hline $\mathrm{K}=N^{2}$ & $\begin{array}{c}\mathrm{n}=5 \\
\text { \# iter. \# }\end{array}$ & $\begin{array}{c}\mathrm{n}=10 \\
\text { iter. }\end{array}$ & $\begin{array}{c}\mathrm{n}=20 \\
\text { \# iter. }\end{array}$ & $\begin{array}{c}\mathrm{n}=40 \\
\text { \# }\end{array}$ \\
\hline 16 & 23 & 25 & 26 & 27 \\
64 & 24 & 26 & 27 & 28 \\
144 & 24 & 26 & 27 & 29 \\
256 & 24 & 26 & 27 & 29 \\
400 & 24 & 26 & 27 & 28 \\
576 & 24 & 26 & 27 & 28 \\
784 & 24 & 26 & 27 & 28 \\
1024 & 23 & 26 & 27 & 28 \\
\hline
\end{tabular}

\begin{tabular}{c|cccc}
\hline $\mathrm{K}=N^{2}$ & $\begin{array}{c}\mathrm{n}=5 \\
\text { \# iter. }\end{array}$ & $\begin{array}{c}\mathrm{n}=10 \\
\text { \# iter. }\end{array}$ & $\begin{array}{c}\mathrm{n}=20 \\
\text { \# }\end{array}$ & $\begin{array}{c}\mathrm{n}=40 \\
\text { iter. }\end{array}$ \\
\hline 16 & 25 & 25 & 27 & 29 \\
64 & 27 & 28 & 30 & 31 \\
144 & 27 & 28 & 30 & 31 \\
256 & 27 & 28 & 30 & 32 \\
400 & 27 & 28 & 30 & 31 \\
576 & 27 & 28 & 30 & 31 \\
784 & 27 & 28 & 30 & 31 \\
1024 & 27 & 28 & 30 & 31 \\
\hline
\end{tabular}

Table 1. Number of conjugate gradient iterations needed for reducing the residual of a factor $10^{-5}$, for different combinations of the number $K=N^{2}$ of subdomains and $n$ elements per edge ( $n^{2}$ elements per subdomains) and for $Q_{1}$ finite elements (left) and $Q_{2}$ finite elements (right).

Acknowledgement. This work was partially supported by the IHP Project Breaking Complexity, contract HPRN-CT-2002-00286.

\section{References}

[AMW99] Achdou, Y., Maday, Y., Widlund, O.: Substructuring preconditioners for the mortar method in dimension two. SIAM J. Numer. Anal., 36, 551-580, (1999)

[BMP94] Bernardi, C., Maday, Y., Patera, A.T.: A new non conforming approach to domain decomposition: The mortar element method. In: Haim Brezis and Jacques-Louis Lions, (ed), Collège de France Seminar. Pitman, (1994)

[BP01] Bertoluzza, S., Perrier, V.: The Mortar Method in the Wavelet Context ESAIM:M2AN, 35, 647-673, (2001)

[BP04] Bertoluzza, S., Pennacchio, M.: Substructuring preconditioners for high order mortar methods. submitted, (2004)

[BPS86] Bramble, J.H., Pasciak, J.E., Schatz, A.H.: The construction of preconditioners for elliptic problems by substructuring, I. Math. Comp., 47, 103-134, (1986)

[W01] Wohlmuth, B.: Discretization Methods and Iterative Solvers Based on Domain Decomposition, volume 17 of Lecture Notes in Computational Science and Engineering. Springer, (2001). 